\title{
Whole genome sequencing of "Faecalibaculum rodentium" ALO17, isolated from C57BL/6J laboratory mouse feces
}

\author{
Sooyeon Lim¹, Dong-Ho Chang ${ }^{1}$, Sharon Ahn ${ }^{1,2}$ and Byoung-Chan Kim ${ }^{1,2^{*}}$
}

\begin{abstract}
Background: Intestinal microorganisms affect host physiology, including ageing. Given the difficulty in controlling for human studies of the gut microbiome, mouse models provide an alternative avenue to study such relationships. In this study, we report on the complete genome of "Faecalibaculum rodentium" ALO17, a bacterium that was isolated from the faeces of a 9-month-old female C57BL/6J mouse. This strain will be utilized in future in vivo studies detailing the relationships between the gut microbiome and ageing.
\end{abstract}

Results: The whole genome sequence of "F. rodentium" ALO17 was obtained using single-molecule, real-time (SMRT) technique on a PacBio instrument. The assembled genome consisted of 2,542,486 base pairs of double-stranded DNA with a GC content of $54.0 \%$ and no plasmids. The genome was predicted to contain 2794 open reading frames, 55 tRNA genes, and 38 rRNA genes. The $16 \mathrm{~S}$ rRNA gene of ALO17 was $86.9 \%$ similar to that of Allobaculum stercoricanis DSM $13633^{\top}$, and the average overall nucleotide identity between strains ALO17 and DSM $13633^{\top}$ was $66.8 \%$. After confirming the phylogenetic relationship between "F. rodentium" ALO17 and A. stercoricanis DSM 13633", their whole genome sequences were compared, revealing that "F. rodentium" ALO17 contains more fermentation-related genes than A. stercoricanis DSM 13633'. Furthermore, "F. rodentium" ALO17 produces higher levels of lactic acid than A. stercoricanis DSM $13633^{\top}$ as determined by high-performance liquid chromatography.

Conclusion: The availability of the "F. rodentium" ALO17 whole genome sequence will enhance studies concerning the gut microbiota and host physiology, especially when investigating the molecular relationships between gut microbiota and ageing.

Keywords: "Faecalibaculum rodentium" ALO17, Whole genome sequencing, Phylogeny, Comparative genomics

\section{Background}

Interactions between animals and their intestinal microorganisms play a crucial role in host physiology [1-3]. For example, perturbations of the intestinal microbiota have been associated with immunological, metabolic, and neurological diseases $[4,5]$. The metabolic activities of intestinal microorganisms directly affect food digestion, absorption, and energy production [6, 7]. The composition of the intestinal microbiota is also

\footnotetext{
*Correspondence: bckim@kribb.re.kr

${ }^{1}$ Microbiomix and Immunity Research Center, Korea Research Institute of Bioscience and Biotechnology (KRIBB), 125 Gwahangno, Yuseong-gu, Daejeon 34141, South Korea

Full list of author information is available at the end of the article
}

related to ageing of the host [8-10]. The gut microbiota changes dramatically between early and late stages of life, with a shift from Lactobacillus and Bifidobacterium to Bacteroidetes and Clostridia genera. This shift suggests a change from lactate metabolism to increased short-chain fatty acid (SCFA) production and carbohydrate metabolism as ageing progresses $[8,10-12]$. To date, multiple studies have investigated the human intestinal microbiome to understand the relationships between the gut microbiota and host ageing $[6,8,9]$. However, difficulties controlling experimental conditions, including diet, medications, and housing status in complex human systems has contributed to inconsistencies in results from such studies. Mouse studies of the 
relationships between the microbiome and host ageing have provided better-controlled systems with consistent results. Langile et al. [10] divided female C57BL/6J mice into three age groups based on murine frailty index (FI) scores and reported that Erysipelotrichaceae was one of the dominant bacterial families colonising the guts of middle-aged mice (589 days old). These data are consistent with our unpublished data from investigations of the microbial diversity in mice of different ages. We found the most abundant operational taxonomic units (OTU) of middle-aged mice (18-21 months old) were related to Allobaculum species [13] within the family Erysipelotrichaceae. Using fresh and anoxic mice faeces and anaerobic culture techniques [13, 14], we isolated a strain closely related (99\% 16S rRNA gene sequence similarity) to the most abundant OTUs from the feces of C57BL/6J mice, and designated it "Faecalibaculum rodentium" ALO17 [13]. However, the overall intestinal microbiota, including the dominant strains present in the guts of different aged mice, has not been investigated in precise detail. In this study, the whole genome sequence of "F. rodentium" ALO17 was generated using a PacBio instrument and compared in silico to the previously-reported genome sequence of Allobaculum stercoricanis DSM $13633^{\mathrm{T}}$.

\section{Methods}

\section{Strain information}

A strictly anaerobic bacterium, ALO17, was previously isolated [13] from the faeces of a 9-month-old female C57BL/6J mouse fed a standard experimental diet (cat. No. 2018S; Harlan Laboratories). The mouse was purchased from DBL Co. Ltd, Korea and housed in the specific pathogen-free facility of Korea Advanced Institute of Science and Technology (KAIST). All animal experiments were performed in accordance with the guidelines and policies for rodent experimentation provided by the Institutional Animal Care and Use Committee (IACUC) of KAIST. This study protocol was approved by the IACUC of KAIST (IACUC-13-140) [13]. The isolate was Gram-stain positive, non-motile, non-spore forming small rod, oxidase and catalase negative. As previously described [13, 15], strictly anaerobic techniques were used for the preparation of the DSM 104 medium (http://www.dsmz.de/microorganisms/medium/pdf/ DSMZ_Medium104.pdf) and the cultivation process (the gas atmosphere was $100 \% \mathrm{~N}_{2}$ ). Strain ALO17 was optimally cultivated in the DSM 104 broth at $37{ }^{\circ} \mathrm{C}$ and at $\mathrm{pH} 7$ for 3 days under strict anaerobic condition. On the basis of polyphasic taxonomic experiments, we have proposed that the isolate be assigned to the family Erysipelothricaceae with the novel genus and species name, "Faecalibaculum rodentium" [13].

\section{Genome sequencing, assembly and annotation}

Genomic DNA was purified from 3L cultures of "F. rodentium" ALO17 as previously described [13, 14]. Extracted DNA samples were sequenced using Pacific Biosciences RS sequencing technology (Pacific Biosciences, Menlo Park, CA), yielding > 50X coverage. Each sample was prepared as a $10-\mathrm{kb}$ insert library using $\mathrm{C} 2$ chemistry and sequenced on the PacBio RS II (Pacific Biosciences), according to the manufacturer's instructions. De novo genome assembly was performed using the CLCbio CLC Genomics Workbench v7.0.4 and PacBio SMRT Analysis 2.2.0 [16]. Annotation was completed using a homology search against the Clusters of Orthologous Groups (COG) and SEED databases $[17,18]$, respectively. SEED viewer [19] was used for subsystem functional categorization of the predicted ORFs and for visualization [20]. Average nucleotide identity (ANI) values were determined using the BLAST algorithm [21].

\section{Comparative genomics}

Comparative genomic analyses were performed using BLAST and a robust pair-wise sequence alignment algorithm. 16S rRNA gene sequences with pairwise similarities $>85 \%$ were obtained from the EZtaxon database (http://www.ezbiocloud.net/eztaxon) for nine different species and used for phylogenetic analyses in MEGA6 [22]. Among the nine strains, whole genome sequences for Eubacterium dolichum DSM3991 ${ }^{\mathrm{T}}$, Faecalitalea cylindroides ATCC $27803^{\mathrm{T}}$, Holdemanella biformis DSM $3989^{\mathrm{T}}$, and Allobaculum stercoricanis DSM $13633^{\mathrm{T}}$ were acquired from the NCBI database (http://www.ncbi.nlm. nih.gov/genome/genomes) and their ANI values [21] to "F. rodentium" ALO17 were calculated. For the calculation of ANI values, the query sequence was randomly cut into fragments of 1020 nucleotides and each was blasted against the subject genome. Following this, a genome tree was constructed using $\mathrm{R}$ software, and the most closely related sequences were determined according to the ANI values using the unweighted pair group method. A phylogenetic tree for the five strains was also generated using MEGA6. Fermentation-related genes in the " $F$. rodentium" ALO17 genome were categorized into functional groups using the annotated genome of $A$. stercoricanis DSM $13633^{\mathrm{T}}$.

\section{Measurements of lactic acid concentration}

The concentration of lactic acid in the anaerobic DSM 104 broth after 3 days cultivation at $37^{\circ} \mathrm{C}$ were determined using an HPLC (1200 Series, Agilent Technology, USA) equipped with an Aminex $87 \mathrm{H}$ column (dimensions: $300 * 7.8$, Bio-Rad, USA). The mobile phase was $0.01 \mathrm{M}$ sulfuric acid with $0.5 \mathrm{ml} / \mathrm{min}$ flow rate at $40{ }^{\circ} \mathrm{C}$. The average values with error ranges of lactic acid 
concentration were obtained from two different duplicate experiments.

\section{Quality assurance}

Highly purified and intact genomic DNA was obtained from 3L cultures grown in the DSM 104 broth by the modified bead-beating technique $[14,23]$ and confirmed against the published genome obtained from the NCBI database. The 16S rRNA gene was extracted from the assembled contigs using the RAST annotation system. ANI values were converted into distances between the other genomes analysed.

\section{Results and discussion}

The genome of " $F$. rodentium" ALO17 as assembled here consisted of a single circular DNA chromosome of 2,542,486 base pairs, a GC content of $54.0 \%$, and no plasmids. The genome contained 2583 predicted open reading frames (ORFs), 55 tRNAs, and 38 rRNAs. Analyses using SEED subsystem categorization and COG functional categorizations are shown in Fig. 1. SEED subsystem categorization predicted 1529 ORFs that encode known functional proteins, whereas 1054 ORFs were of unknown function. Among the ORFs with a predicted function, 274 were predicted to be for carbohydrate synthesis, 200 were predicted to be for amino acid synthesis, 191 for protein metabolism, 140 for RNA metabolism, 122 for DNA metabolism, and 78 for the production of cofactors, vitamins, prosthetic groups, and pigments. Additionally, 40 ORFs belonged to the fermentation category. There are five major roles for genes in this category. A total of 13 ORFs (32.5\%) were similar to genes responsible for the fermentation of acetyl-CoA to butyrate, 12 (30.0\%) were predicted to be involved in fermentations with mixed acids, 9 (22.5\%) in butanol biosynthesis, $4(10.0 \%)$ in lactate fermentation, and $2(5.0 \%)$ were predicted to be acetolactate synthase subunits. COG analyses assigned 1566 ORFs (91.0\% of all predicted ORFs) to functional categories. Among these, 815 ORFs (47.39\% of the COG-assigned ORFs) belonged to 5 primary categories: 204 ORFs belonged to Category L (replication, recombination, and repair), 182 to Category $\mathrm{G}$ (carbohydrate transport and metabolism), 146 to Category E (amino acid transport and metabolism), 142 to Category K (transcription), and 141 to Category J (translation, ribosomal structure and biogenesis). For comparative genomic analyses, two methods were used: ANI and 16S rRNA gene sequencing. Nine bacterial species with pairwise similarities $>85 \%$ for the $16 \mathrm{~S}$ rRNA gene, compared to "F. rodentium" ALO17, were selected. Among those selected, whole genome sequences for four species were in the NCBI database. A phylogenetic tree was constructed based on the 16S rRNA gene sequences. ANI values calculated between ALO17, E. dolichum DSM3991 ${ }^{\mathrm{T}}$, F. cylindroides ATCC $27803^{\mathrm{T}}, H$. biformis DSM $3989^{\mathrm{T}}$, A. stercoricanis DSM $13633^{\mathrm{T}}$, were 63.9, 65.8, 66.2 and $66.8 \%$, respectively. Strain ALO17 clustered with strain DSM $13633^{\mathrm{T}}$ in the phylogenetic tree, which was supported by a high bootstrap value and ANI dendrogram (Fig. 2). Due to their high level of relatedness, additional detailed genomic analyses were performed between " $F$. rodentium" ALO17 and other 4 reference of A. stercoricanis DSM $13633^{\mathrm{T}}, H$. biformis DSM $3989^{\mathrm{T}}, F$. cylindroides ATCC $27803^{\mathrm{T}}$ and E. dolichum DSM $3991^{\mathrm{T}}$. This comparison of the fermentation related genes is summarised in Table 1 . " $F$. rodentium" ALO17 had a single homologue predicted to encode a protein that stimulates sugar/maltose fermentation. Additionally, subsystems for acetolactate synthase subunits, the fermentation of acetyl-CoA to butyrate, butanol biosynthesis, lactate fermentation, and mixed acid fermentation were present in both " $F$. rodentium" ALO17 and $A$. stercoricanis DSM $13633^{\mathrm{T}}$ genomes. The numbers of genes in " $F$. rodentium" ALO17 and A. stercoricanis DSM $13633^{\mathrm{T}}$ predicted to be involved in fermentation subsystems were 40 and 23, respectively. " $F$. rodentium" ALO17 had more genes predicted to be involved in specific functions, compared to A. stercoricanis DSM $13633^{\mathrm{T}}$. Such genes were predicted to encode for 3-hydroxybutyryl-CoA dehydrogenase (EC 1.1.1.157), 3-hydroxybutyryl-CoA dehydrogenase (EC 1.1.1.157), electron transfer flavoprotein (alpha and beta subunit), 3-hydroxybutyryl-CoA dehydrogenase (EC 1.1.1.157), pyruvate formate-lyase (EC 2.3.1.54), L-lactate dehydrogenase (EC 1.1.1.27), pyruvate formate-lyase (EC 2.3.1.54), and pyruvate formate-lyase activating enzyme (EC1.97.1.4). These data suggest that this isolate has stronger fermentation activity than $A$. stercoricanis DSM $13633^{\mathrm{T}}$. To test this hypothesis, the lactic acid concentrations were measured in the growth medium of " $F$. rodentium" ALO17 and A. stercoricanis DSM $13633^{\mathrm{T}}$ by high-performance liquid chromatography. After three days of incubation at $37{ }^{\circ} \mathrm{C}$, the lactic acid concentrations were observed to be $9.5 \pm 0.6 \mathrm{mM}$ (strain ALO17) and $4.9 \pm 0.8 \mathrm{mM}$ (A. stercoricanis DSM $13633^{\mathrm{T}}$ ), respectively. Previous study of $A$. stercoricanis DSM $13633^{\mathrm{T}}$ showed the level of lactic acid was $3.5 \mathrm{mM}$ [24]. In summary, despite their phylogenetic clustering, " $F$. rodentium" ALO17 differed from A. 
$\mathbf{a}$

COG distribution

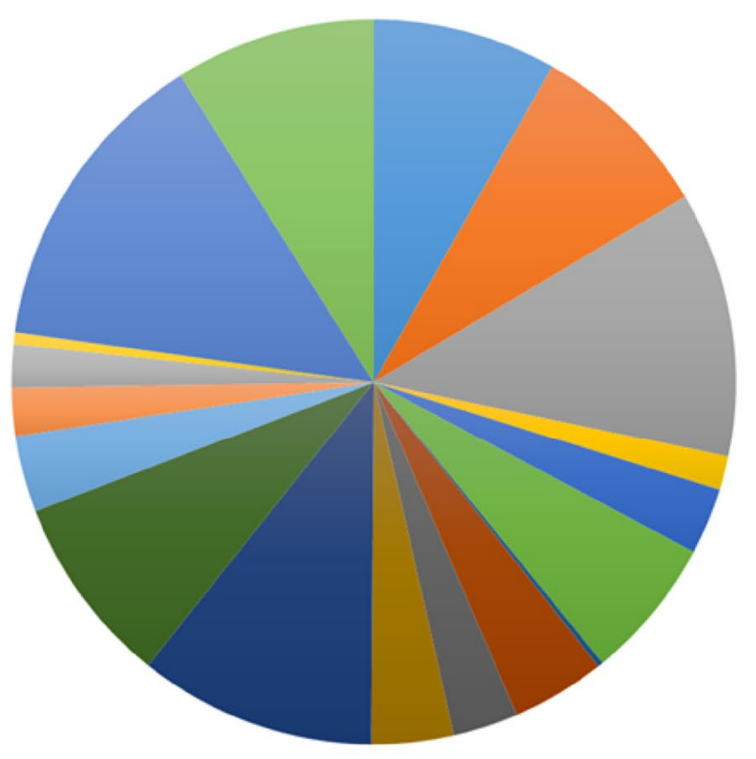

b

Subsystem distribution

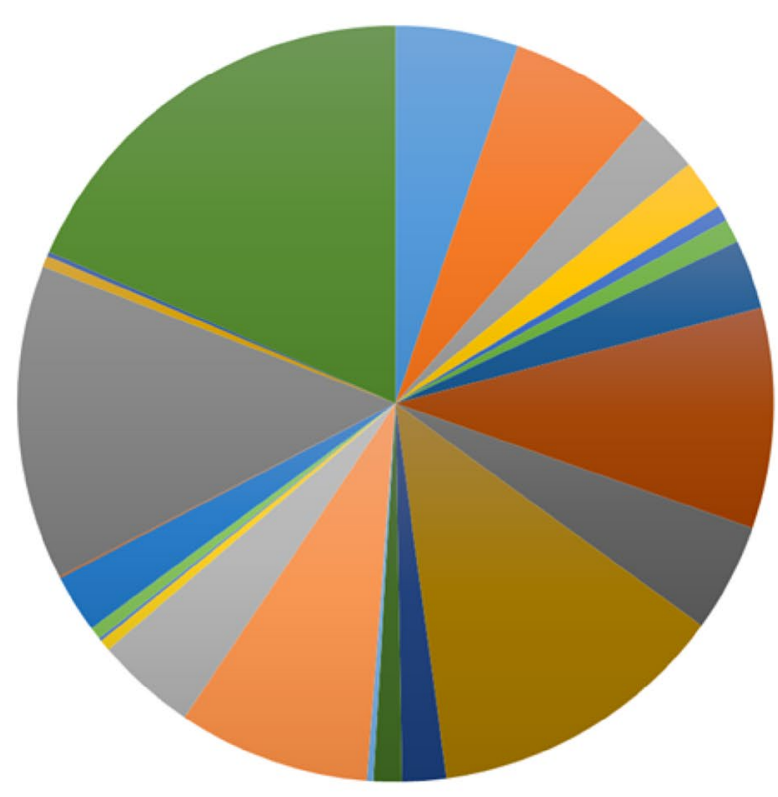

COG category feature (counts)

$=$ Translation, ribosomal structure and biogenesis (141)

= Transcription (142)

$=$ Replication, recombination and repair (204)

$=$ Cell cycle control, cell division, chromosome partitioning (26)

= Posttranslational modification, protein turnover, chaperones (51)

\# Cell wall/membrane/envelope biogenesis (109)

= Cell motility (4)

= Inorganic ion transport and metabolism (72)

= Signal transduction mechanisms (50)

= Energy production and conversion (63)

a Carbohydrate transport and metabolism (182)

= Amino acid transport and metabolism (146)

= Nucleotide transport and metabolism (59)

= Coenzyme transport and metabolism (37)

= Lipid transport and metabolism (33)

= Secondary metabolites biosynthesis, transport and catabolism (9)

$=$ General function prediction only (238)

= Function unknown (154)

\section{Subsystem feature (counts)}

॥ Cofactors, Vitamins, Prosthetic Groups, Pigments (78)

= Cell Wall and Capsule (92)

= Virulence, Disease and Defense (40)

= Potassium metabolism (32)

- Miscellaneous (10)

= Phages, Prophages, Transposable elements, Plasmids (15)

- Membrane Transport (44)

= RNA Metabolism (140)

= Nucleosides and Nucleotides (69)

= Protein Metabolism (191)

- Cell Division and Cell Cycle (28)

- Regulation and Cell signaling (18)

= Secondary Metabolism (4)

- DNA Metabolism (122)

$=$ Fatty Acids, Lipids, and Isoprenoids (64)

= Nitrogen Metabolism (7)

= Dormancy and Sporulation (2)

nespiration (8)

- Stress Response (37)

= Metabolism of Aromatic Compounds (1)

= Amino Acids and Derivatives (200)

= Sulfur Metabolism (7)

a Phosphorus Metabolism (9)

= Carbohy drates (274)

Fig. 1 Statistics of annotated genes for "F. rodentium" Alo17 based on a COG and b SEED databases 
a

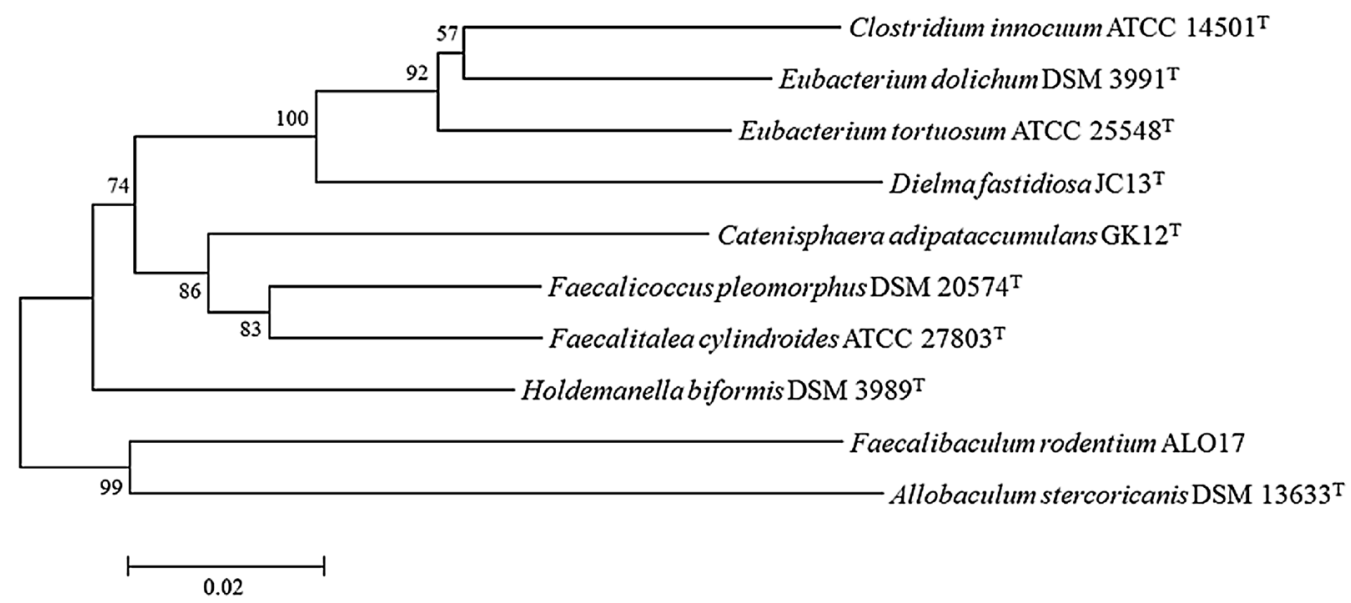

b

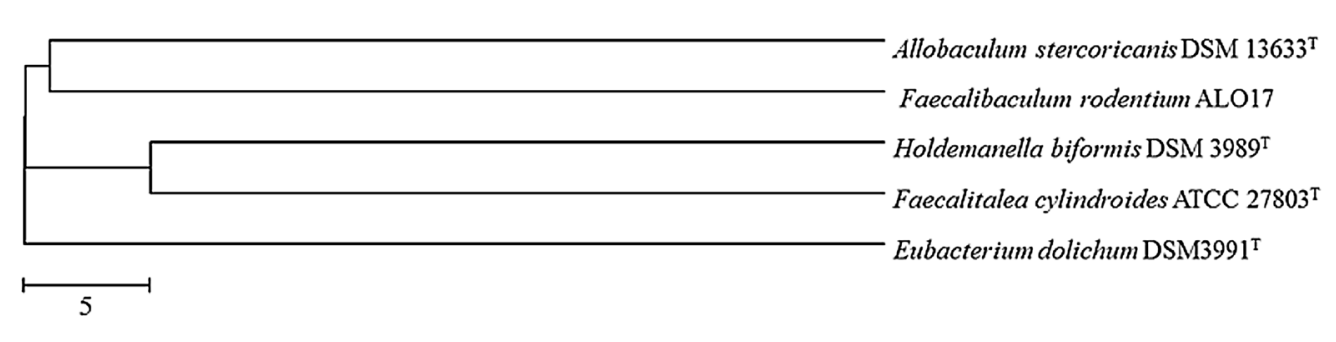

Allobaculum stercoricanis DSM $13633^{\mathrm{T}}$

$\mathrm{b}$

c

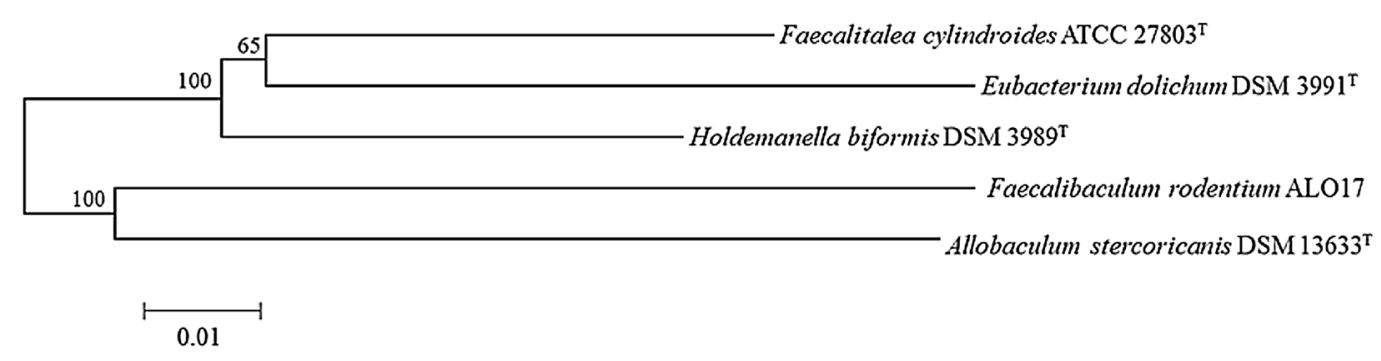

Fig. 2 a Phylogenetic tree analysis of 10 strains using 165 rRNA sequence method (pairwise similarity $>85 \%$ ). b Phylogenetic tree analysis of 5 strains using ANI (average nucleotide identity) methods. c Phylogenetic tree analysis of 5 strains using 165 rRNA sequence method. Bootstrap values (expressed as percentages of 1000 replication, $>50 \%$ ) are shown at branching points. Bar 0.02 substitution per nucleotide position

stercoricanis DSM $13633^{\mathrm{T}}$ in lactic acid production. We hypothesize that " $F$. rodentium" ALO17 is an obligate anaerobe that replaces Lactobacilli and Bifidobacterium in middle-aged mice. Lactobacilli and Bifidobacterium are primary lactic acid producers in young mice; however, as the gut becomes strictly anaerobic with age, " $F$. rodentium" may become dominant. Thus, dominance of lactate producers and lactate production in the animal gut might be inversely related to ageing [10].

\section{Initial findings}

The genome of "F. rodentium" ALO17 contained 2583 predicted open reading frames (ORFs), 55 tRNAs, and 38 rRNAs. Among them, 40 and 23 genes in " $F$. 
Table 1 Comparison of fermentation related organism between "F. rodentium" ALO17 and other 4 references of $A$. stercoricanis DSM $13633^{\top}$, H. biformis DSM $3989^{\top}$, F. cylindroides ATCC $27803^{\top}$ and E. dolichum DSM $3991^{\top}$

\begin{tabular}{|c|c|c|c|c|c|c|}
\hline \multirow[t]{2}{*}{ Subsystem } & \multirow[t]{2}{*}{ Role description } & \multicolumn{5}{|c|}{ Number of genes } \\
\hline & & Alo17 & $13633^{\top}$ & $3989^{\top}$ & $27803^{\top}$ & $3991^{\top}$ \\
\hline Butanol biosynthesis & Alcohol dehydrogenase (EC 1.1.1.1) & 1 & 1 & 1 & 1 & 1 \\
\hline Butanol biosynthesis & NADH-dependent butanol dehydrogenase A (EC 1.1.1.-) & 1 & 1 & 1 & 0 & 1 \\
\hline Butanol biosynthesis & Pyruvate formate-lyase (EC 2.3.1.54) & 3 & 1 & 5 & 4 & 1 \\
\hline Butanol biosynthesis & 3-Hydroxybutyryl-CoA dehydrogenase (EC 1.1.1.157) & 3 & 1 & 1 & 1 & 1 \\
\hline Butanol biosynthesis & Acetyl-CoA acetyltransferase (EC 2.3.1.9) & 1 & 1 & 2 & 2 & 1 \\
\hline Fermentations: mixed acid & Alcohol dehydrogenase (EC 1.1.1.1) & 1 & 1 & 1 & 1 & 1 \\
\hline Fermentations: mixed acid & L-lactate dehydrogenase (EC 1.1.1.27) & 2 & 1 & 2 & 2 & 1 \\
\hline Fermentations: mixed acid & Sugar/maltose fermentation stimulation protein homolog & 1 & 0 & 0 & 0 & 0 \\
\hline Fermentations: mixed acid & Pyruvate formate-lyase (EC 2.3.1.54) & 3 & 1 & 5 & 4 & 1 \\
\hline Fermentations: mixed acid & Pyruvate formate-lyase activating enzyme (EC 1.97.1.4) & 3 & 1 & 4 & 4 & 1 \\
\hline Fermentations: mixed acid & Phosphate acetyltransferase (EC 2.3.1.8) & 1 & 1 & 1 & 0 & 1 \\
\hline Fermentations: Mixed acid & Acetate kinase (EC 2.7.2.1) & 1 & 1 & 1 & 1 & 1 \\
\hline Acetolactate synthase subunits & Acetolactate synthase large subunit (EC 2.2.1.6) & 1 & 1 & 0 & 0 & 0 \\
\hline Acetolactate synthase subunits & Acetolactate synthase small subunit (EC 2.2.1.6) & 1 & 1 & 0 & 0 & 0 \\
\hline Fermentations: lactate & L-Lactate dehydrogenase (EC 1.1.1.27) & 2 & 1 & 2 & 2 & 1 \\
\hline Fermentations: lactate & Phosphate acetyltransferase (EC 2.3.1.8) & 1 & 1 & 1 & 0 & 1 \\
\hline Fermentations: lactate & Acetate kinase (EC 2.7.2.1) & 1 & 1 & 1 & 1 & 1 \\
\hline Acetyl-CoA fermentation to butyrate & 3-Hydroxybutyryl-CoA dehydratase (EC 4.2.1.55) & 1 & 1 & 1 & 1 & 0 \\
\hline Acetyl-CoA fermentation to butyrate & Electron transfer flavoprotein, beta subunit & 2 & 1 & 1 & 1 & 0 \\
\hline Acetyl-CoA fermentation to butyrate & Electron transfer flavoprotein, alpha subunit & 2 & 1 & 1 & 1 & 0 \\
\hline Acetyl-CoA fermentation to butyrate & 3-Hydroxybutyryl-CoA dehydrogenase (EC 1.1.1.157) & 3 & 1 & 1 & 1 & 0 \\
\hline Acetyl-CoA fermentation to butyrate & 3-Hydroxyacyl-CoA dehydrogenase (EC 1.1.1.35) & 3 & 1 & 1 & 1 & 0 \\
\hline Acetyl-CoA fermentation to butyrate & Acetyl-CoA:acetoacetyl-CoA transferase, alpha subunit (EC 2.8.3.8) & 1 & 1 & 2 & 1 & 0 \\
\hline Acetyl-CoA fermentation to butyrate & Acetyl-CoA Acetyltransferase (EC 2.3.1.9) & 1 & 1 & 2 & 2 & 0 \\
\hline
\end{tabular}

rodentium" ALO17 and A. stercoricanis DSM $13633^{\mathrm{T}}$ predicted to be involved in fermentation subsystems. This result suggest that " $F$. rodentium" ALO17 has more fermentation activity than $A$. stercoricanis DSM $13633^{\mathrm{T}}$. The lactic acid concentrations were measured by highperformance liquid chromatography and " $F$. rodentium" ALO17 produces higher levels of lactic acid than A. stercoricanis DSM $13633^{\mathrm{T}}$.

\section{Future directions}

This is the first report on the complete genome sequence of " $F$. rodentium" ALO17. This bacterium was isolated from a 9-month-old laboratory mouse and its genome was sequenced using PacBio SMRT technology. " $F$. rodentium" ALO17 is phylogenetically related to $A$. stercoricanis DSM $13633^{\mathrm{T}}$, which belongs to the family Erysipelotrichaceae, and this family of bacterium is dominant in the gut of middle-aged mice [10]. Considering the robust production of lactic acid in this isolate, further analyses will provide useful information regarding the relationships between gut microbiota, lactate metabolism, and host ageing.

\section{Availability of supporting data}

The genome sequence of " $F$. rodentium" ALO17 was deposited in the Genbank under the accession number of CP011391.

\section{Authors' contributions}

SY Lim and BC Kim designed the study. DH Chang and SR Ahn performed the experiments. SY Lim analyzed and conducted whole genome sequencing comparison. All authors read and approved the final manuscript.

\section{Author details}

${ }_{1}^{1}$ Microbiomix and Immunity Research Center, Korea Research Institute of Bioscience and Biotechnology (KRIBB), 125 Gwahangno, Yuseong-gu, Daejeon 34141, South Korea. ${ }^{2}$ Department of Biosystems and Bioengineering, University of Science and Technology (UST), 217 Gwahangno, Yuseong-gu, Daejeon, South Korea.

\section{Acknowledgements}

This work was supported by the Research Program for Agricultural Science and Technology Development (Project No. PJ010168) and was partially supported by Grants from the National Research Foundation of Korea (NRF) 
(2008-2004721 and NRF-2013M3A9A5076601 and 2015M3C9A4053394), the KRIBB Research Initiative Programs (KGS4121551) and by a Grant from of the Korea Health Technology R\&D Project (HI14C0368).

\section{Competing interests}

The authors declare that they have no competing interests.

Received: 17 September 2015 Accepted: 1 February 2016 Published online: 12 February 2016

\section{References}

1. Vaughan EE, de Vries MC, Zoetendal EG, Ben-Amor K, Akkermans AD, de Vos WM. The intestinal LABs. Antonie Van Leeuwenhoek. 2002;82(1-4):341-52.

2. Hooper LV, Wong MH, Thelin A, Hansson L, Falk PG, Gordon J. Molecular analysis of commensal host-microbial relationships in the intestine. Science. 2001;291(5505):881-4.

3. Sommer F, Backhed F. The gut microbiota [mdash] masters of host development and physiology. Nat Rev Micro. 2013;11(4):227-38.

4. Su CG, Judge TA, Lichtenstein GR. Extraintestinal manifestations of inflammatory bowel disease. Gastroenterol Clin North Am. 2002;31(1):307-27.

5. Mazmanian SK, Liu CH, Tzianabos AO, Kasper DL. An immunomodulatory molecule of symbiotic bacteria directs maturation of the host immune system. Cell. 2005;122(1):107-18.

6. Ley RE, Turnbaugh PJ, Klein S, Gordon Jl. Microbial ecology: human gut microbes associated with obesity. Nature. 2006;444(7122):1022-3.

7. Nicholson JK, Holmes E, Kinross J, Burcelin R, Gibson G, Jia W, Pettersson S. Host-gut microbiota metabolic interactions. Science. 2012;336(6086):1262-7.

8. Yatsunenko T, Rey FE, Manary MJ, Trehan I, Dominguez-Bello MG, Contreras M, Magris M, Hidalgo G, Baldassano RN, Anokhin AP. Human gut microbiome viewed across age and geography. Nature. 2012;486(7402):222-7.

9. Lozupone CA, Stombaugh J, Gonzalez A, Ackermann G, Wendel D, Vázquez-Baeza Y, Jansson JK, Gordon JI, Knight R. Meta-analyses of studies of the human microbiota. Genome Res. 2013;23(10):1704-14.

10. Langille MG, Meehan CJ, Koenig JE, Dhanani AS, Rose RA, Howlett SE, Beiko RG. Microbial shifts in the aging mouse gut. Microbiome. 2014;2(1):50.
11. van Tongeren SP, Slaets JP, Harmsen H, Welling GW. Fecal microbiota composition and frailty. Appl Environ Microbiol. 2005;71(10):6438-42.

12. Mariat D, Firmesse $O$, Levenez F, Guimarăes V, Sokol H, Dore J, Corthier $\mathrm{G}$, Furet J. The Firmicutes/Bacteroidetes ratio of the human microbiota changes with age. BMC Microbiol. 2009;9(1):123.

13. Chang D-H, Rhee M-S, Ahn S, Bang B-H, Oh JE, Lee HK, Kim B-C. "Faecalibaculum rodentium" gen. nov., sp. nov., isolated from the faeces of a laboratory mouse. Antonie Van Leeuwenhoek. 2015;108(Pt6):1309-18.

14. Lee J-H, Kumar S, Lee G-H, Chang D-H, Rhee M-S, Yoon M-H, Kim B-C. Methanobrevibacter boviskoreani sp. nov., isolated from the rumen of Korean native cattle. Int J Syst Evol Microbiol. 2013;63(Pt 11):4196-201.

15. Bang B-H, Rhee M-S, Chang D-H, Park D-S, Kim B-C. Erysipelothrix larvae sp. nov., isolated from the larval gut of the rhinoceros beetle, Trypoxylus dichotomus (Coleoptera: Scarabaeidae). Antonie Van Leeuwenhoek. 2015;107(2):443-51.

16. Chin C-S, Alexander DH, Marks P, Klammer AA, Drake J, Heiner C, Clum A, Copeland A, Huddleston J, Eichler EE. Nonhybrid, finished microbial genome assemblies from long-read SMRT sequencing data. Nat Methods. 2013;10(6):563-9.

17. Tatusov RL, Koonin EV, Lipman DJ. A genomic perspective on protein families. Science. 1997;278(5338):631-7.

18. Disz T, Akhter S, Cuevas D, Olson R, Overbeek R, Vonstein V, Stevens R, Edwards RA. Accessing the SEED genome databases via Web services API: tools for programmers. BMC Bioinformatics. 2010;11(1):319.

19. Overbeek R, Olson R, Pusch GD, Olsen GJ, Davis JJ, Disz T, Edwards RA, Gerdes S, Parrello B, Shukla M. The SEED and the rapid annotation of microbial genomes using subsystems technology (RAST). Nucleic Acids Res. 2014;42(D1):D206-14.

20. Aziz RK, Bartels D, Best AA, DeJongh M, DiszT, Edwards RA, Formsma K, Gerdes S, Glass EM, Kubal M. The RAST server: rapid annotations using subsystems technology. BMC Genom. 2008;9(1):75.

21. Goris J, Konstantinidis KT, Klappenbach JA, Coenye T, Vandamme P, Tiedje JM. DNA-DNA hybridization values and their relationship to wholegenome sequence similarities. Int J Syst Evol Microbiol. 2007;57(1):81-91.

22. Tamura K, Stecher G, Peterson D, Filipski A, Kumar S. MEGA6: molecular evolutionary genetics analysis version 6.0. Mol Biol Evol. 2013;30(12):2725-9.

23. Fujimoto $S$, Nakagami Y, Kojima F. Optimal bacterial DNA isolation method using bead-beating technique. Memoirs Kyushu Univ Dep Of Health Scis Of Medical Sch. 2004;3:33-8.

24. Greetham HL, Gibson GR, Giffard C, Hippe H, Merkhoffer B, Steiner U, Falsen E, Collins MD. Allobaculum stercoricanis gen. nov., sp. nov., isolated from canine feces. Anaerobe. 2004;10(5):301-7.

\section{Submit your next manuscript to BioMed Central and we will help you at every step:}

- We accept pre-submission inquiries

- Our selector tool helps you to find the most relevant journal

- We provide round the clock customer support

- Convenient online submission

- Thorough peer review

- Inclusion in PubMed and all major indexing services

- Maximum visibility for your research

Submit your manuscript at www.biomedcentral.com/submit

\section{() Biomed Central}

